\title{
MFS ANALYSIS OF THE VIBRATION FILTERING EFFECT OF PERIODIC STRUCTURES IN ELASTIC MEDIA
}

\author{
LUÍS GODINHO $^{1}$, PAULO AMADO-MENDES ${ }^{1}$, PEDRO ALVES-COSTA $^{2}$ \& CARLOS ALBINO ${ }^{1}$ \\ ${ }^{1}$ ISISE, Department Civil Engineer, University of Coimbra, Rua Luis Reis Santos, Coimbra, Portugal. \\ ${ }^{2}$ Construct-FEUP, Department Civil Engineer, Faculty of Engineering of the University of Porto, R. \\ Dr. Roberto Frias, Porto, Portugal.
}

\begin{abstract}
Phononic crystals have been extensively studied, and their capacity to attenuate the propagation of sound waves at specific frequency bands is well known and documented in the literature. However, few studies exist concerning the behaviour of such structures in the context of elastic media, with the purpose of attenuating the transmission of vibrations. Applying this concept can be quite interesting, and may allow new vibration control devices to be developed, tailored at specific applications. As an example, buried periodic structures may be used to control elastic wave propagation in the ground, and thus to help reducing the vibrations that can reach sensible structures.

In this work, the authors make use of a 2.5D numerical model based on the Method of Fundamental Solutions (MFS) to analyse this complex problem, considering the case of arrays of elastic inclusions buried in a homogeneous medium, fully considering the complete elastodynamic interaction between the inclusions and the host medium. Due to the geometric periodicity of the analysed problem, the numerical formulation can be simplified, particularly in what concerns the calculation of the system matrix, and significant computational gains can be obtained. The results of a numerical study concerning the behaviour of a sequence of embedded inclusions within an elastic material, when subject to the incidence of waves with different frequencies, is here presented, and the interpretation of the involved phenomena is described in order to clarify the main wave propagation features in the presence of multiple elastic inclusions. The computed results are promising, clearly revealing the existence of band gaps where large attenuation occurs, although limitations related to the existence of guided waves traveling along the inclusions are also identified.

Keywords: elastodynamics, MFS, periodic structures, vibration filtering
\end{abstract}

\section{INTRODUCTION}

Many important problems, such as those related to human comfort, make vibration mitigation a relevant worldwide societal challenge. New generation of mitigation measures include the so-called metamaterials, which, in essence, are artificial materials engineered to have properties not directly found in nature. Among these metamaterials, periodically arranged arrays of scatterers constitute a particular case, usually designated as "phononic crystals" or "sonic crystals". Application of these metamaterials has been tried in acoustic shielding, as described, for example, by Umnova et al. [1] or by Martinez-Sala et al. [2]. Many of the published works in that field indicate that these techniques can be effective, and seem to be quite promising for acoustical applications. These results also make it possible to expect that similar concepts may be used with success in vibration mitigation using buried metamaterial-like structures, as can be the case, for example, of buried sonic-crystal-like structures.

Recent works concerning "seismic" protection by periodic structures are already found in the literature. Kim and Das [3] analysed the effect of a periodic structure in the creation of a shadow zone to seismic waves. Alagoz and Alagoz [4] presented numerical studies concerning the effect of what they called "seismic crystals", indicating an effective attenuation of seismic waves. Possibly the first experimental evidence that buried periodic structures can efficiently attenuate vibrations in specific frequency bands is due to Brûle et al. [5]; these authors have shown experimental results of a seismic test carried out using seismic waves 
generated by a monochromatic vibrocompaction probe, revealing a modification of the seismic energy distribution in the presence of a buried metamaterial, in agreement with numerical simulations. Preliminary work by the authors of the present paper has also demonstrated the attenuating properties of arrays of scatterers (see [6]), although in a very limited number of scenarios. It looks like this concept for vibration protection based on buried metamaterials may constitute a powerful promising future solution.

Beyond the physical phenomena themselves, one of the challenges of this type of problem is related to their accurate computational simulation. Previous works in the field of wave propagation (as for example [7,8]) made use of a number of techniques, which can be extended and adapted to treat the problem of metamaterials in solid media. Methods such as the BEM (Boundary Element Method) or the MFS (Method of Fundamental Solutions) seem to be appropriate choices for that purpose. Conceptually, one possibility is related to $2.5 \mathrm{D}$ analysis, in which the geometry remains constant along one direction, although with a purely 3D source. These models [9] are very efficient and can be of interest to study the propagation of elastic waves in the presence of arrays of scatterers; they may serve as an excellent base for understanding the underlying physics of such complex phenomena.

The present paper intends to provide a numerical strategy based on the use of a 2.5D MFS implementation, and tries to physically interpret the wave propagation phenomena that occur around solid buried inclusions embedded in a host homogeneous soil. Due to the geometric periodicity and geometrically repetitive nature of the analysed problem, the numerical implementation can be significantly simplified, particularly in what concerns the calculation of the system matrix; indeed, a large number of repeated blocks occur in this matrix, whose calculation just needs to be performed once, and so significant computational gains can be obtained. A number of numerical simulations is here used to illustrate the main features of wave propagation in this type of scenarios, using different arrays of scatterers and considering different elastic properties.

\section{MATHEMATICAL FORMULATION}

\subsection{5D Problem Formulation}

The problem to be solved consists of an elastic medium within which a set of elastic bodies is embedded, composing a multi-domain elastic system. For this type of problem, the frequency domain wave equation can be defined as

$$
(\lambda+2 \mu) \nabla \nabla \cdot \mathbf{u}-\mu \nabla \times \nabla \times \mathbf{u}+\omega^{2} \rho \mathbf{u}=0
$$

In this equation, $\mathbf{u}$ is the displacement vector, $\lambda$ and $\mu$ are the Lamé's constants and $\rho$ is the mass density of the elastic medium.

Consider now that in this physical system the solid inclusions have a constant geometry along the $z$ direction, and the whole system is subjected to a harmonic point source, which is oscillating with an angular frequency $\omega$. Since the system's geometry is infinite and does not change along the $z$ direction, it is possible to apply a spatial Fourier transformation along that direction and to determine the 3D displacement field as a continuous integral of simpler bidimensional solutions, as

$$
\mathbf{u}^{3 D}=\int_{-\infty}^{+\infty} \mathbf{u}^{2.5 D}(k) \mathrm{e}^{-\mathrm{i} k\left(z-z_{0}\right)} d k
$$


where $k$ is the axial wavenumber. Discretising this integral and rewriting this equation as a discrete summation (assuming an infinite set of virtual sources equally spaced $L_{v s}$ along the axial direction, such that $\Delta k_{z}=2 \pi / L_{v s}$ ), the 3D displacement field may be written as

$$
\mathbf{u}^{3 D}=\sum_{m=-\infty}^{+\infty} \mathbf{u}^{2.5 D}\left(k_{z}\right) \mathrm{e}^{-\mathrm{i} k_{z}\left(z-z_{0}\right)} \Delta k_{z} \mathrm{e}^{-\mathrm{i} k_{z}\left(z-z_{0}\right)}
$$

where $k_{z}=m \Delta k_{z}$. This summation converges, and can be approximated by a finite number of terms, ranging from $-M$ to $+M$. Note that, in order to avoid any spatial contamination from the virtual sources, the distance $L_{v s}$ must be sufficiently large, and that the case with $k_{z}=0$ corresponds to the $2 \mathrm{D}$ problem.

\subsection{General formulation of the Method of Fundamental Solutions (MFS)}

The MFS approximates the solution in a given elastic medium in terms of a linear combination of fundamental solutions of the governing differential equation. In fact, a set of $N S$ fictitious sources is located outside the elastic domain (namely at points $X_{0}^{n}$, with $n=1 \ldots N S$ ), in order to circumvent singularities, and then the displacement field at any point of the host medium can be written as a summation of the contribution of those virtual loads, with a-priori unknown amplitudes $A_{j}^{n}$. Therefore, the displacement $u_{i}$, at a generic point $X$, along direction $i=x, y, z$ is represented by:

$$
u_{i}\left(X, k_{z}, \omega\right)=\sum_{n=1}^{N S} \sum_{j=1}^{3} G_{i j}\left(X, X_{0}^{n}, k_{z}, \omega\right) A_{j}^{n}\left(k_{z}, \omega\right)
$$

where $G_{i j}$ corresponds to the displacement generated at point $X$, along direction $i$, by a unit load acting along direction $j$ at point $X_{0}^{n}$, which can be determined by using the appropriate fundamental solutions.

This approach allows the incorporation of different Green's functions without introducing any significant changes to the method; thus it is quite simple to use Green's functions that account, for example, for the presence of a half-space or of a layered medium. For the simpler case of an infinite homogeneous elastic medium (full-space), the required Green's functions $G_{i j}\left(X, X_{0}^{n}, k_{z m}, \omega\right)$ for displacements in the different directions, with $i, j=x, y, z$, were presented by Tadeu and Kausel [10], and can be shortly defined as:

$$
\begin{gathered}
G_{x x}\left(X, X_{0}, k_{z}, \omega\right)=\frac{1}{4 \mathrm{i} \rho \omega^{2}}\left[k_{s}^{2} H_{0 \beta}-\frac{1}{r} B_{1}+\gamma_{x}^{2} B_{2}\right] \\
G_{y y}\left(X, X_{0}, k_{z}, \omega\right)=\frac{1}{4 \mathrm{i} \rho \omega^{2}}\left[k_{s}^{2} H_{0 \beta}-\frac{1}{r} B_{1}+\gamma_{y}^{2} B_{2}\right] \\
G_{z z}\left(X, X_{0}, k_{z}, \omega\right)=\frac{1}{4 \mathrm{i} \rho \omega^{2}}\left[k_{s}^{2} H_{0 \beta}-k_{z}^{2} B_{0}\right] \\
G_{x y}\left(X, X_{0}, k_{z}, \omega\right)=G_{y x}\left(X, X_{0}, k_{z}, \omega\right)=\gamma_{x} \gamma_{y} \frac{1}{4 \mathrm{i} \rho \omega^{2}} B_{2} \\
G_{x z}\left(X, X_{0}, k_{z}, \omega\right)=G_{z x}\left(X, X_{0}, k_{z}, \omega\right)=\mathrm{i} k_{z} \gamma_{x} \frac{1}{4 \mathrm{i} \rho \omega^{2}} B_{1}
\end{gathered}
$$




$$
G_{y z}\left(X, X_{0}, k_{z}, \omega\right)=G_{z y}\left(X, X_{0}, k_{z}, \omega\right)=\mathrm{i} k_{z} \gamma_{y} \frac{1}{4 \mathrm{i} \rho \omega^{2}} B_{1}
$$

with $B_{n}=k_{\beta}^{n} H_{n \beta}-k_{\alpha}^{n} H_{n \alpha}, k_{\alpha}=\sqrt{k_{p}^{2}-k_{z}^{2}}$ with $\operatorname{Im} k_{\alpha}<0, k_{p}=\omega / \alpha, k_{\beta}=\sqrt{k_{s}^{2}-k_{z}^{2}}$ with $\operatorname{Im} k_{\beta}<0, k_{s}=\omega / \beta, \alpha$ and $\beta$ are the $\mathrm{P}$ and $\mathrm{S}$ wave propagation velocities, $\gamma_{l}=\partial r / \partial x_{l}=x_{l} / r$ with $l=x, y, H_{n \alpha}=H_{n}^{(2)}\left(k_{\alpha} r\right), H_{n \beta}=H_{n}^{(2)}\left(k_{\beta} r\right)\left(H_{n}^{(2)}\right.$ is the Hankel function of second type and $n^{\text {th }}$ order) and $r$ stands for the distance between the source and field points.

To define a solution methodology for the specific problem in which elastic inclusions are embedded in a host infinite medium, let us illustrate the formulation of the method for the case of a single inclusion (Fig. 1). For that case, it becomes necessary to define a number of discrete points along the solid-solid interface where the necessary boundary conditions need to be enforced (collocation points). Two sets of virtual sources need also to be defined, one of them located within the inclusion that allows simulation of the outer field, and the other surrounding the inclusion in order to simulate the wave field inside it. Using equn (4), the corresponding displacement fields can thus be calculated.

In the present case, boundary conditions imply continuity of displacements and equilibrium of tractions along the boundary between the two distinct domains. Imposing those conditions, a linear equation system with $2 \times 3 \times N S$ equations and $2 \times 3 \times N S$ unknowns is established, with the form

$$
\left[\begin{array}{c}
\mathbf{G}_{(1)}\left(k_{z}, \omega\right)-\mathbf{G}_{(2)}\left(k_{z}, \omega\right) \\
\mathbf{H}_{(1)}\left(k_{z}, \omega\right)-\mathbf{H}_{(2)}\left(k_{z}, \omega\right)
\end{array}\right] \mathbf{A}\left(k_{z}, \omega\right)=\left[\begin{array}{c}
-\mathbf{u}_{i n c}\left(k_{z}, \omega\right) \\
-\tilde{i n c}\left(k_{z}, \omega\right)
\end{array}\right]
$$

where $\mathbf{G}_{(\mathrm{k})}$ and $\mathbf{H}_{(\mathrm{k})}$ stand for coefficients matrices related to medium $\mathrm{k}$, assembled following the definition of terms $G_{i j}$ and their combined derivatives together with the corresponding constitutive law, respectively; A stands for the unknown amplitude vector; and $\mathbf{u}_{i n c}$ and $\tilde{i n c}$, incorporate the displacements and tractions originated by a real source at the collocation points, respectively.

Solution of this equation system allows obtaining the unknown amplitude coefficients of the virtual sources, which in turn allow computing the relevant wave fields inside and outside the inclusions. The process illustrated here for a single inclusion can easily be generalized for multiple inclusions.

\subsection{Implementation details for periodic geometries}

If a set of $\mathrm{N}$ inclusions is considered, equally spaced between them forming a regular matrix, the calculation of the MFS system matrix can be greatly accelerated. Indeed, for that case, a

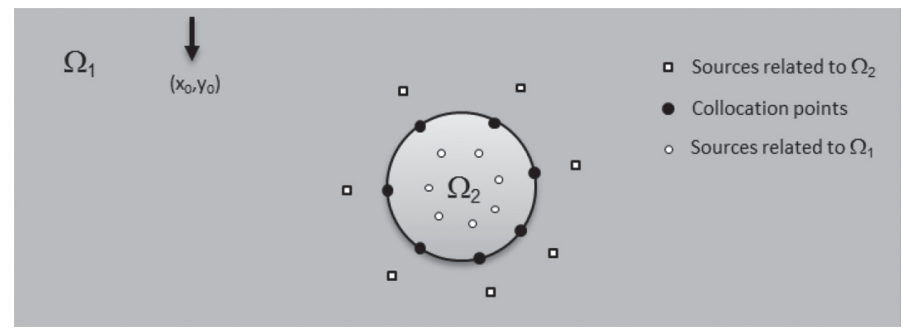

Figure 1: Schematic representation of the MFS model. 
more general form of equn (6) must be used, in which each block itself is built as a sequence of smaller blocks. For example for $\mathbf{G}_{(1)}\left(k_{z}, \omega\right)$ and $\mathbf{G}_{(2)}\left(k_{z}, \omega\right)$, the corresponding form can be written as

$$
\begin{array}{r}
\mathbf{G}_{(1)}\left(k_{z}, \omega\right)=\left[\begin{array}{cccc}
\mathbf{G}_{(1)}^{1,1}\left(k_{z}, \omega\right) & \mathbf{G}_{(1)}^{1,2}\left(k_{z}, \omega\right) & \cdots & \mathbf{G}_{(1)}^{1, N}\left(k_{z}, \omega\right) \\
\mathbf{G}_{(1)}^{2,1}\left(k_{z}, \omega\right) & \mathbf{G}_{(1)}^{2,2}\left(k_{z}, \omega\right) & \cdots & \mathbf{G}_{(1)}^{2, N}\left(k_{z}, \omega\right) \\
\vdots & \vdots & \ddots & \vdots \\
\mathbf{G}_{(1)}^{N, 1}\left(k_{z}, \omega\right) & \mathbf{G}_{(1)}^{N, 2}\left(k_{z}, \omega\right) & \cdots & \mathbf{G}_{(1)}^{N, N}\left(k_{z}, \omega\right)
\end{array}\right] \\
\mathbf{G}_{(2)}\left(k_{z}, \omega\right)=\left[\begin{array}{cccc}
\mathbf{G}_{(2)}^{1,1}\left(k_{z}, \omega\right) & \mathbf{0} & \cdots & \mathbf{0} \\
\mathbf{0} & \mathbf{G}_{(2)}^{2,2}\left(k_{z}, \omega\right) & \cdots & \mathbf{0} \\
\vdots & \vdots & \ddots & \vdots \\
\mathbf{0} & \mathbf{0} & \cdots & \mathbf{G}_{(2)}^{N, N}\left(k_{z}, \omega\right)
\end{array}\right]
\end{array}
$$

In each block, the $i, j$ superscript indicates the effect of the set of sources related to inclusion $j$ on the set of collocation points around inclusion $i$; when the internal field of each inclusion is considered $\left(\mathbf{G}_{(2)}\left(k_{z}, \omega\right)\right)$, a large number of null matrix blocks occurs, since the set of sources around the inclusion only influences its internal field.

It is important to notice that, if the inclusions are periodically placed, many of the blocks of $\mathbf{G}_{(1)}\left(k_{z}, \omega\right)$ are repeated, and may only be calculated once; this can be easily realized, for example, for all diagonal blocks. Although this behaviour has been described for the . $\mathbf{G}_{(1)}\left(k_{z}, \omega\right)$. submatrix, it is equally applicable to $\mathbf{H}_{(1)}\left(k_{z}, \omega\right)$.

Given this observation, a practical implementation algorithm to compute $\mathbf{G}_{(1)}\left(k_{z}, \omega\right)$ and $\mathbf{H}_{(1)}\left(k_{z}, \omega\right)$ can simply be written as:

- The calculation starts, looping $i$ and $j$ between 1 and $N$ :

- The vector connecting the centers of scatterer $i$ and $j$ is computed $\left(\mathbf{v}=\mathbf{x}_{i}^{S C}-\mathbf{x}_{j}^{S C}\right)$, and compared to the ones calculated in previous loop steps.

* If two blocks connected by $\mathbf{v}$ have been previously calculated, then there is no need to recalculate the block $i, j$, and the reference of the previously calculated block is associated to $i, j$;

* Otherwise, block $i, j$ is calculated and stored, and vector $\mathbf{v}$ is added to the list of already calculated blocks;

- At the end of this loop, all necessary blocks are calculated.

\section{MODEL VERIFICATION AND PERFORMANCE}

To first check the correctness of the proposed model and of its implementation, a homogeneous system is simulated, for which the analytical solution corresponds to the fundamental solutions described above. Using the proposed strategy, a matrix with $3 \times 3$ elastic inclusions, with radius $0.3 \mathrm{~m}$, equally spaced $1.2 \mathrm{~m}$ is considered to be embedded in a host medium, and the same properties are ascribed to both the host medium and to the inclusions. These properties correspond to a Young modulus of $100 \mathrm{MPa}$, a density of $2000 \mathrm{~kg} / \mathrm{m}^{3}$ and a Poisson's ratio of 0.2 . A source illuminates the system at $x=-2.0$ and $y=0.0$, vertically centred with the inclusions, and responses are computed at a receiver positioned at $x=5.3$ and $y=1.8$. Figure 2 illustrates the computed displacements at the receiver, originated by a vertical load, together with the analytical solution, and considering an apparent velocity of $1336 \mathrm{~m} / \mathrm{s}$ (defined as $c_{z}=\omega / k_{z}$ ). The presented plots clearly evidence an excellent match between the two results. 

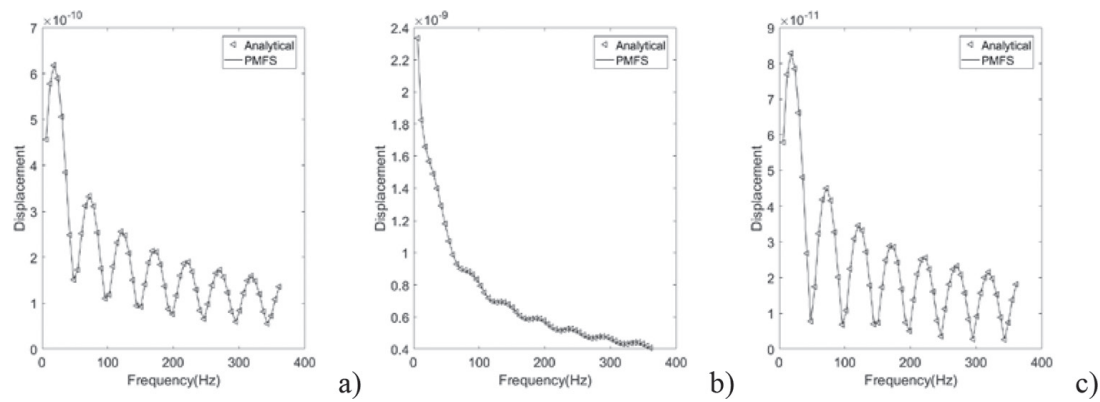

Figure 2: Verification of the implemented algorithm for $\mathrm{x}(\mathrm{a}), \mathrm{y}(\mathrm{b})$ and $\mathrm{z}(\mathrm{c})$ displacements.

To understand the performance gain between the proposed scheme (designated by PMFS) and a traditional MFS implementation, a sequence of tests has been performed, recording the CPU times required by each approach. Geometries with $4 \times 4,5 \times 5,6 \times 6$ and $7 \times 7$ inclusions are considered, with 30 collocation points describing each inclusion; a maximum MFS matrix size of $8820 \times 8820$ is thus obtained for the larger problem. The internal material of the inclusions is assumed to have a Young modulus of $30 \mathrm{GPa}$, a density of $2500 \mathrm{~kg} / \mathrm{m}^{3}$ and a Poisson's ratio of 0.3 . Figure 3 illustrates the performance of the methods using a bar chart, in which each bar is divided in two parts: the lower (dark grey) part is related to the CPU time required for matrix formation; the upper (lighter grey) is related to the solution of the equation system. Since the same solver is used in both cases, the required CPU time for solution of the equation system is always the same for the two schemes. Analysing the plot, it is clear that a significant reduction of the CPU time occurs for the proposed implementation, which is related only to the matrix formation time. Comparing the dark grey parts of the plotted bars, this reduction is very large, and in general only $20 \%$ to $30 \%$ of the CPU time is required.

\section{NUMERICAL APPLICATIONS}

The presented model has been used to study the wave propagation patterns and the physical behaviour of a system composed by different numbers of inclusions. A number of results is shown for different geometries, composed of arrangements of circular cylinders. All results are presented in terms of normalized slowness and of normalized frequency (nondimensional

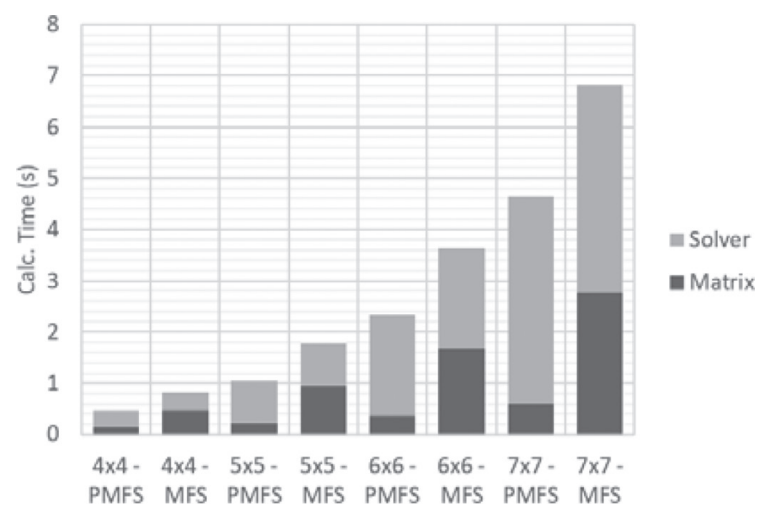

Figure 3: CPU times for the proposed (PMFS) and for the classical MFS implementations. 
parameters). The normalized frequency is given in terms of the relation between the lattice constant of the arrangement of cylinders (spacing between consecutive centres) and the wavelength of the $\mathrm{S}$ waves in the solid host medium, allowing to define:

$$
\bar{F}=a / \lambda_{s}=a \times f / c_{s}
$$

The normalized slowness is defined as the relation of the axial wavenumber $\left(\mathrm{k}_{\mathrm{z}}\right)$ to the $\mathrm{S}$ wave wavenumber, given as:

$$
k=\frac{k_{z}}{\omega / c_{s}}
$$

The following material properties are used in the present work:

In the presented results, different geometrical arrangements are analysed, including both square and triangular lattice distributions. The typical arrangement for each test case is represented in Fig. 4, for examples with $2 \times 5$ inclusions in a rectangular lattice, and for $2 \times 3$ inclusions in a triangular lattice.

The first set of examples presented here is related to the case in which perfectly rigid and unmovable inclusions are considered, embedded in a solid medium with the properties of $\mathrm{M} 1$, where $\mathrm{S}$ waves propagate at $144.3 \mathrm{~m} / \mathrm{s}$, and $\mathrm{P}$ waves at $235.7 \mathrm{~m} / \mathrm{s}$.

Figure 5 presents the amplitude level of the combined $x, y$ and $z$ velocities at $\mathrm{R} 1$. It is clear that for normalized slownesses above 1, very little energy propagates in the system, and, in

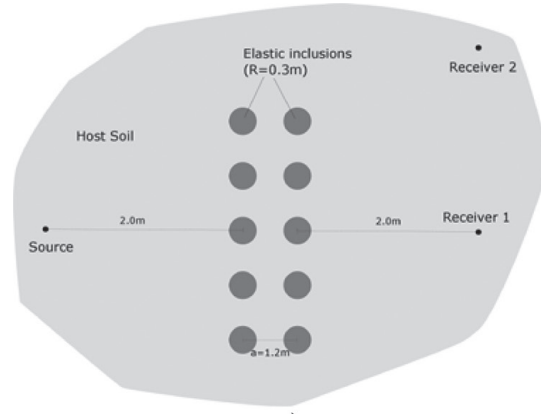

a)

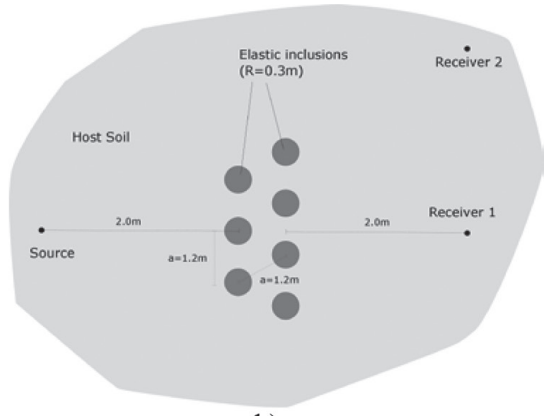

b)

Figure 4: Illustrative scheme of the tested configurations for $2 \times 5$ square (a) and $2 \times 3$ triangular (b) lattices.
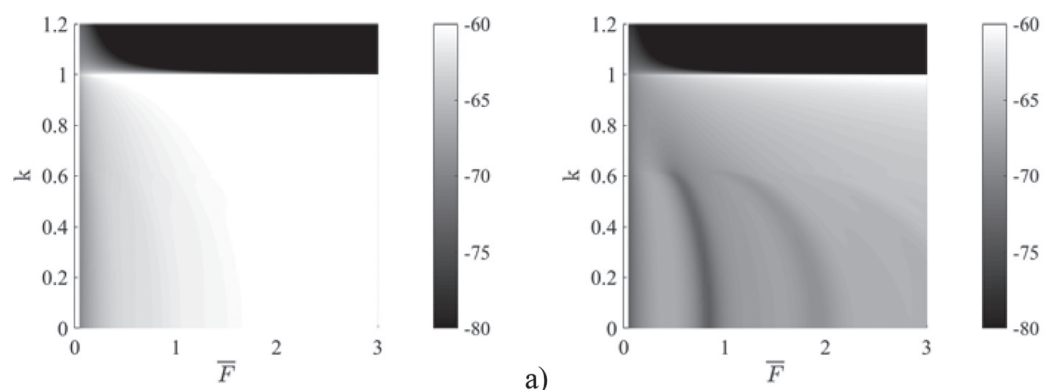

a)

b)

Figure 5: Absolute vibration velocity levels at R1 for an homogeneous medium (a) and in the presence of a single rigid inclusion (b). 
fact, only evanescent waves exist. Bellow this slowness, since the elastic waves propagate freely, without obstacles, increasing vibration velocities are seen as the frequency increases. This scenario greatly changes in the presence of a rigid inclusion, as can be seen in Fig. 5b (where a rigid inclusion of radius $0.3 \mathrm{~m}$ is considered between the source and the receiver). Here, a significant decrease in the velocity levels is observed throughout the whole analysed $k-\bar{F}$ domain, since the presence of the obstacle blocks the direct incidence of waves at the receiver.

To understand the effect of multiple inclusions, disposed in an organized pattern defined by a lattice constant $a$, in the wave propagation pattern, several tests were performed for different scenarios. To facilitate the interpretation of the effect of the multiple inclusions, the next plots illustrate the results in terms of "insertion loss" (IL), given by the difference of vibration levels registered at the receiver without and with the intermediate structures.

Figure 6 shows the IL computed for an array of 6 inclusions, disposed in a rectangular manner, considering a lattice constant $\mathrm{a}=1.2 \mathrm{~m}$ and an inclusion radius of $0.3 \mathrm{~m}$. The structure of the presented plots has now become much more complex, and includes several features. Indeed, multiple interactions occur between inclusions, originating constructive and destructive interferences. However, one particular feature merits special attention, particularly the presence of a high attenuation frequency range visible as a pattern of lighter shades in the presented plots. This zone occurs for the full sequence of $k$ values and indicates that a strong attenuation is originated by the presence of the set of inclusions. This effect occurs mostly between normalized frequencies of 0.5 and 1.5 , that is, between $f=0.5 c_{s} / a$ and $f=1.5 c_{s} / a$ (this zone is marked with 1 in the plot). Interestingly, this lower limit can be related to the theory of the acoustic attenuation by sonic crystal, in which the band gap frequency can usually be defined as $f=0.5 c_{s} / a$; thus, it can be inferred that, possibly, the dominant effect controlling the energy attenuation by this system is the same as that observed in acoustics. An additional effect that is also seen in the presented plots is related to a constructive interference between the multiple inclusions (these regions are marked with 2 in the plot).

Figure 7 illustrates similar plots computed for triangular shaped inclusion distributions, considering $3 \times 3$ (total of 10) inclusions and $5 \times 3$ (total of 17) inclusions. The plots presented in this figure reveal a quite similar behaviour to those in Fig. 6, although some differences can be observed. Indeed, the "band-gap" identified above seems to be now more marked and narrower, even for the $3 \times 3$ case, and seems to become progressively more intense when more inclusions are added. For the case of 10 inclusions, the "band-gap" effect is indeed very well defined, and corresponds to a continuous bright patch in the Fig. $7 \mathrm{~b}$, occurring between normalized frequencies of 0.5 and 1.0. Additionally, it becomes clear that one of the constructive effects identified above (the leftmost one marked " 2 " in Fig. 6b) has now
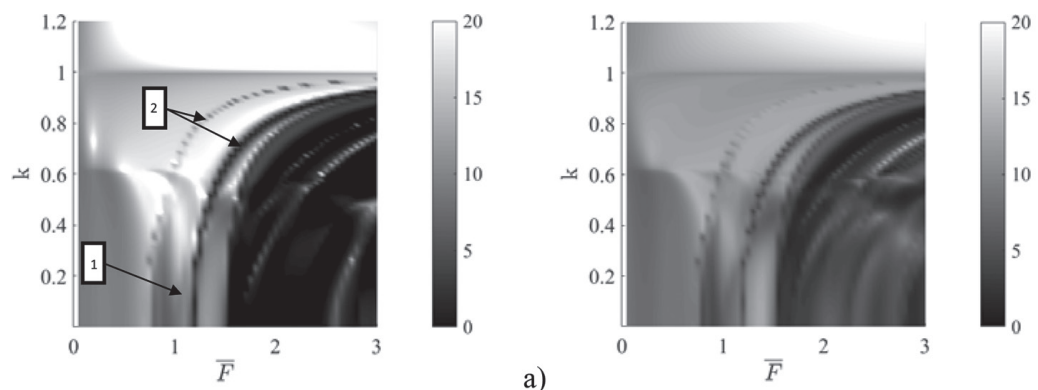

b)

Figure 6: Insertion Loss at R1 (a) and R2 (b) for a 3×3 square lattice system. 

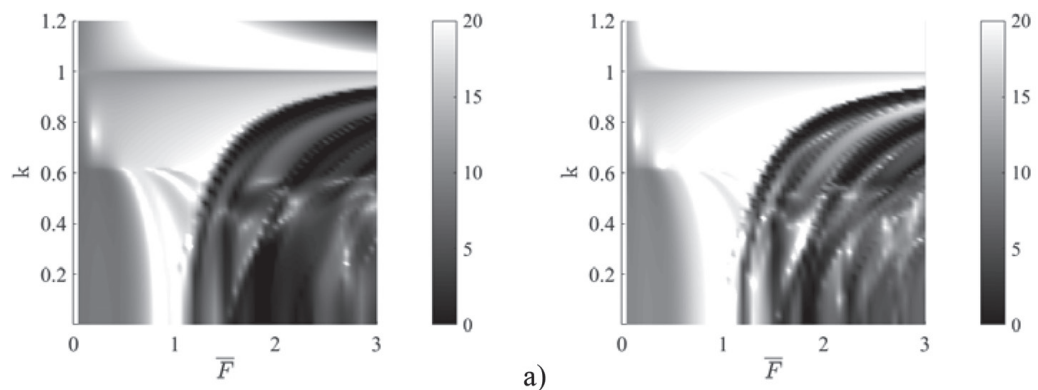

Figure 7: Insertion Loss at R1 for triangular lattices considering $3 \times 3$ (a) and $5 \times 3$ (b) groups.

disappeared; that effect can be related to the diagonal distance between inclusions in the rectangular distribution, which is eliminated when triangular lattices are considered.

To understand the effect of considering the inclusions as being made of an elastic material, simulations were performed for the geometrical configuration used in Fig. $7 \mathrm{~b}(5 \times 3$ triangular lattice). Two different material properties were considered for the inclusions, namely those indicated for M2 and M3 in Table 1. Figure 8 illustrates the IL at receiver 1 for both materials.

Observing the plotted results, it is clear that new features appear due to the consideration of the elastic properties of the inclusions. Indeed, although the upper part of the plot remains essentially unchanged, there is a very distinct behaviour on the lower part. This behaviour may be associated with the longitudinal vibration modes of the inclusions, namely their flexural and torsional modes, which allow a significant part of the energy to pass through and

Table 1: Material properties

\begin{tabular}{llll}
\hline Material & Density $\left(\mathbf{k g} / \mathbf{m}^{\mathbf{3}}\right)$ & E $(\mathbf{M P a})$ & Poisson \\
\hline M1 & 2000 & 100 & 0.2 \\
M2 & 7800 & 200000 & 0.3 \\
M3 & 2500 & 30000 & 0.3 \\
\hline
\end{tabular}

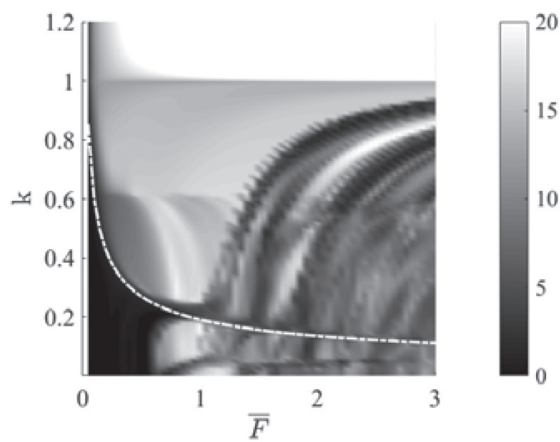

a)

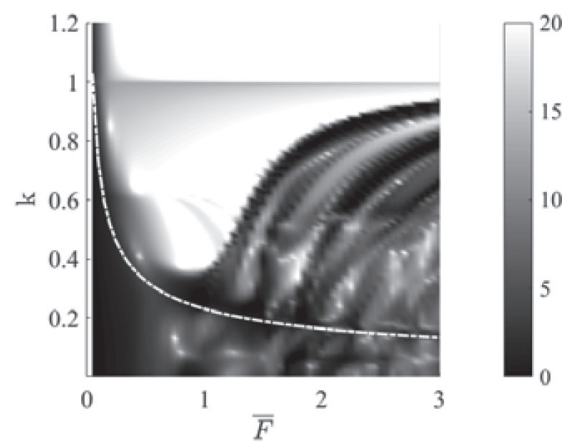

b)

Figure 8: Insertion Loss at R1 when the inclusions are made of M2 (a) and M3 (b). 

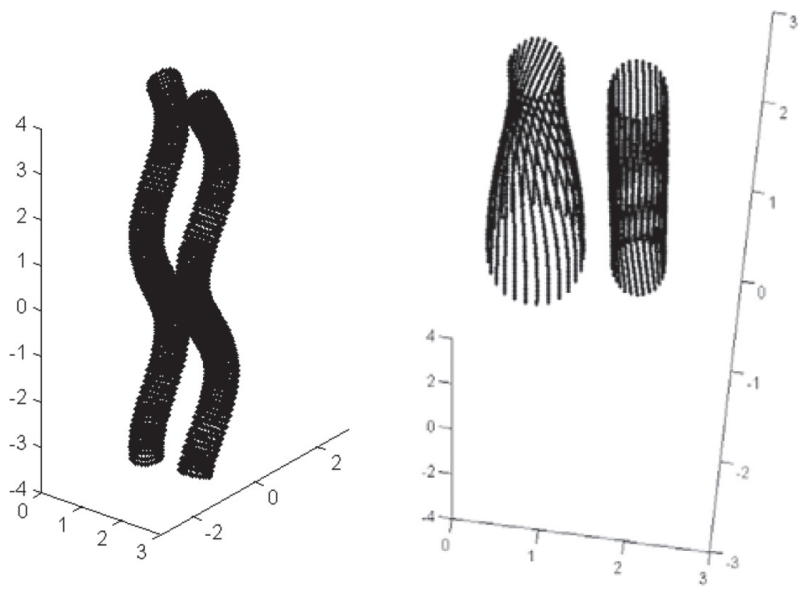

Figure 9: Deformed configuration of elastic inclusions computed for $k-\bar{F}$ pairs corresponding to the bending wave (left) and torsional wave (right) dispersion curves.

reach the receivers. To confirm this hypothesis, the dispersion curve for longitudinal bending waves has been computed for both material types, and is represented in the plots by a dashed line. Clearly, this new line divides the upper and lower parts of the plot, and below it, a completely different behaviour is seen when compared with the plots depicted in Figure 6 or 7 . For lower values of $\mathrm{k}$, the additional contribution of a torsional mode is seen, which also contributes to a reduced IL observed here. To confirm that these are indeed the referred modes, the deformed configuration of the inclusions has been calculated for $k-\bar{F}$ pairs located over the corresponding dispersion curves, and is depicted in Fig. 9. To facilitate the interpretation, only two inclusions are plotted. Observing those plots, it can be seen that a clear bending behaviour is observed on the left plot, while a torsional behaviour is seen on the right plot, thus confirming the described effects.

The observations made above for the case of elastic inclusions allow inferring that accounting for the correct elastic behaviour of the inclusions is essential, since the global behaviour is very different from that seen in the case of a rigid inclusion. The elastic behaviour of the inclusions seems to clearly decrease the attenuation efficiency of the periodic structures, mainly for lower values of the normalized slowness.

\section{CONCLUSIONS}

In the present paper, a study regarding the simulation of the dynamic behaviour of an elastic sonic-crystal-like structure, accounting for its 3D behaviour. The proposed model is based in a 2.5D elastodynamic formulation, making use of an improved version of the Method of Fundamental Solutions specifically tailored for periodic structures. It was shown that the proposed model is quite adequate and very efficient for the analysis of such structures, and allows simulations to be performed even for quite large systems.

Some application examples were presented regarding the analysis of that type of problems, and interesting conclusions could be drawn. It can be said that the simulated arrays of rigid cylinders essentially present the expected behaviour of a sonic-crystal, exhibiting a clear band-gap. The position of this band-gap can be approximately estimated considering 
the $\mathrm{S}$ wave velocity, in a similar manner to that used in acoustic problems. When square and triangular lattices were compared, the latter seem to exhibit a smoother and cleaner behaviour, with a very well defined band-gap region, although affecting a narrower frequency band. Finally, it was observed that when elastic inclusions are considered, a dramatic change occurs, and the vibrational behaviour of the inclusions introduces a very significant degradation of the IL, particularly for low values of the normalized slowness (k), below the dispersion curve associated with the bending waves of the inclusions. This behaviour represents a significant issue that must be addressed in order to efficiently use this concept for vibration mitigation.

\section{ACKNOWLEDGEMENTS}

The authors acknowledge the financial support of FCT - Foundation for Science and Technology and COMPETE, through research project PTDC/ECM-COM/1364/2014. This work is also financed by FEDER funds through the Competitivity Factors Operational Programme - COMPETE and by national funds through FCT - Foundation for Science and Technology within the scope of the projects POCI-01-0145-FEDER-007633 (ISISE) and POCI-01-0145FEDER-007457 (CONSTRUCT).

\section{REFERENCES}

[1] Umnova, O., Attenborough, K. \& Linton, C.M., Effects of porous covering on sound attenuation by periodic arrays of cylinders. The Journal of the Acoustical Society of Amercia, 119(1), pp. 278-284, 2006.

https://doi.org/10.1121/1.2133715

[2] Martinez-Sala, R., Rubio, C., Garcia-Raffi, L., Sanchez-Perez, J.V., Sanchez-Perez, E.A. \& Llinares, J., Control of noise by trees arranged like sonic crystals. Journal Sound Vibration, 291(100), pp. 100-106, 2006. https://doi.org/10.1016/j.jsv.2005.05.030

[3] Kim, S.H. \& Das, M.P., Artificial seismic shadow zone by acoustic metamaterials. Modern Physics Letters B, 27(20), pp. 1350140, 2013. https://doi.org/10.1142/s0217984913501406

[4] Alagoz, B.B. \& Alagoz, S., Towards earthquake shields: a numerical investigation of earthquake shielding with seismic crystals. Open Journal of Acoustics, 1(03), p. 63, 2011. https://doi.org/10.4236/oja.2011.13008

[5] Brûlé, S., Javelaud, E.H., Enoch, S. \& Guenneau, S., Experiments on seismic metamaterials: molding surface waves. Physical Review Letters, 112(13), pp. 133901, 2014. https://doi.org/10.1103/physrevlett.112.133901

[6] Amado-Mendes, P. \& Godinho, L., Reduction of vibrations transmitted through the soil by multiple buried inclusions-numerical analysis. ICOVP - 11th International Conference on Vibration Problems (in CD-Rom), 2013.

[7] Martins, M., Godinho, L. \& Picado-Santos, L., Numerical evaluation of sound attenuation provided by periodic structures. Archives of Acoustics, 38(4), pp. 503-516, 2013. https://doi.org/10.2478/aoa-2013-0060

[8] Santos, P.G., Carbajo, J., Godinho, L. \& Ramis, J., Sound propagation analysis on sonic crystal elastic structures using the Method of Fundamental Solutions (MFS). Computers, Materials and Continua, 43(2), pp. 109-136, 2014.

https://doi.org/10.3970/cmc.2014.043.109 
[9] Godinho, L. \& Tadeu, A., The importance of a small wall deformation in the threedimensional acoustic logging results. Geophysical Journal International, 151(2), pp. 403-415, 2002. https://doi.org/10.1046/j.1365-246X.2002.01792.x

[10] Tadeu, A.J. \& Kausel, E., Green's functions for two-and-a-half-dimensional elastodynamic problems. Journal of Engineering Mechanics, 126(10), pp. 1093-1097, 2000. https://doi.org/10.1061/(ASCE)0733-9399(2000)126:10(1093) 